\title{
Improved Limits on Spin-Mass Interactions
}

\author{
Junyi Lee, Attaallah Almasi, and Michael Romalis \\ Department of Physics, Princeton University, Princeton, New Jersey 08544, USA
}

(Received 9 January 2018; published 17 April 2018)

\begin{abstract}
Very light particles with $C P$-violating couplings to ordinary matter, such as axions or axionlike particles, can mediate long-range forces between polarized and unpolarized fermions. We describe a new experimental search for such forces between unpolarized nucleons in two $250 \mathrm{~kg} \mathrm{~Pb}$ weights and polarized neutrons and electrons in a ${ }^{3} \mathrm{He}-\mathrm{K}$ comagnetometer located about $15 \mathrm{~cm}$ away. We place improved constraints on the products of scalar and pseudoscalar coupling constants, $g_{p}^{n} g_{s}^{N}<4.2 \times 10^{-30}$ and $g_{p}^{e} g_{s}^{N}<1.7 \times 10^{-30}\left(95 \%\right.$ C.L.) for axionlike particle masses less than $10^{-6} \mathrm{eV}$, which represents an order of magnitude improvement over the best previous neutron laboratory limit.
\end{abstract}

DOI: 10.1103/PhysRevLett.120.161801

Introduction.-Spin-mass interactions between spinpolarized fermions and unpolarized fermions can arise in several ways. A particularly well-known and theoretically motivated mechanism arises from the exchange of an axion, a light pseudo Nambu-Goldstone pseudoscalar boson [1,2] associated with a spontaneously broken Peceei-Quinn $U(1)$ symmetry that was originally postulated to solve the strong $C P$ problem in QCD [3]. Its existence is of particular interest since it could potentially explain the unnaturally small level of $C P$ violation in QCD $[4,5]$ and also make up a significant fraction of the dark matter in the Universe [6]. In addition to the QCD axion, other light axionlike particles (ALPs) can arise from the breaking of additional $U(1)$ symmetries, which for example, happens naturally in string theory [7-9].

The spin-mass interaction mediated by a pseudoscalar particle involves a scalar and a pseudoscalar coupling, the strengths of which are parametrized by the dimensionless coupling constants $g_{s}$ and $g_{p}$, respectively. At tree level, this interaction yields the following "monopole-dipole" potential in the nonrelativistic limit [10],

$$
V(\mathbf{r})=\frac{\hbar^{2} g_{s} g_{p}}{8 \pi m_{p}} \hat{r} \cdot \hat{\sigma_{p}}\left(\frac{m_{\phi} c}{\hbar r}+\frac{1}{r^{2}}\right) e^{-r m_{\phi} c / \hbar},
$$

where $m_{p}$ is the mass of the fermion at the pseudoscalar vertex and $\hat{\sigma_{p}}$ is the normalized expectation value of its spin. $\hat{r}$ is the unit direction vector between the particles, $r$ is their separation distance, and $m_{\phi}$ is the mass of the axion. For the QCD axion, its mass and the strength of its fermion couplings are related to each other by the QCD anomaly, while for

Published by the American Physical Society under the terms of the Creative Commons Attribution 4.0 International license. Further distribution of this work must maintain attribution to the author(s) and the published article's title, journal citation, and DOI. Funded by SCOAP .
ALPs they can be considered as independent parameters. One can also construct models involving a very light spin-1 $Z^{\prime}$ boson that generates a long-range spin-mass force similar to Eq. (1) if it has $C P$-violating couplings [11,12].

Searches for axions and axionlike particles have been conducted using a variety of methods. If they constitute a substantial fraction of dark matter and have electromagnetic couplings, they can be detected by photon conversion in a strong magnetic field [13]. Irrespective of dark matter content, axions have been searched for in emission from the Sun [14], photon-axion-photon conversion "light shining through wall" experiments [15], and by looking for longrange forces other than gravitational and electromagnetic. The long-range force experiments can be classified into mass-mass [16], spin-mass [17-23] and spin-spin experiments $[24,25]$, which constrain different combinations of $g_{s}$ and $g_{p}$ couplings. Astrophysical observations also place a number of constraints on axion parameters [26]. Several new axion search methods have been recently proposed and new experiments are being developed [27,28].

Here we report the results of a new search for long-range forces using a $\mathrm{K}-{ }^{3} \mathrm{He}$ comagnetometer and a movable unpolarized source mass. The comagnetometer measures the difference between interactions of electron spin in $\mathrm{K}$ and nuclear (primarily neutron) spin in ${ }^{3} \mathrm{He}$. Barring accidental cancellation, we set new limits on both neutron and electron couplings, improving laboratory limits in the axion mass range near $10^{-6} \mathrm{eV}$.

Experimental apparatus.-The basic operating principles of a $\mathrm{K}^{3} \mathrm{He}$ comagnetometer are described in more detail in [29]. Briefly, the comagnetometer uses overlapping ensembles of spin-polarized $\mathrm{K}$ and ${ }^{3} \mathrm{He}$, which are strongly coupled via their Fermi-contact interaction during spin-exchange collisions [30] when the resonant frequencies of $\mathrm{K}$ and ${ }^{3} \mathrm{He}$ are matched [31]. This coupling gives the comagnetometer fast transient response and 
automatic cancellation of magnetic fields in all three directions.

$\mathrm{K}$ and ${ }^{3} \mathrm{He}$ atoms are contained in a spherical glass-blown cell made from GE180 glass with a diameter of $15 \mathrm{~mm}$ filled with $11 \mathrm{~atm}$. of ${ }^{3} \mathrm{He}$ (at room temperature), 20 Torr of $\mathrm{N}_{2}$ for quenching, and a droplet of $\mathrm{K}$ and ${ }^{87} \mathrm{Rb}$ alkali metal. The ${ }^{87} \mathrm{Rb}$ is introduced in small quantities to enable hybrid optical pumping [32], which allows one to create a uniform alkali polarization in an optically thick cell [33]. At the cell operating temperature of $198^{\circ} \mathrm{C}$ the density of $\mathrm{Rb}$ is measured to be $2 \times 10^{12} \mathrm{~cm}^{-3}$ and the density of $\mathrm{K}$ is $1 \times 10^{14} \mathrm{~cm}^{-3}$. Under typical operating conditions ${ }^{87} \mathrm{Rb}$ is polarized to $70 \%$ by optical pumping with $500 \mathrm{~mW}$ of $795 \mathrm{~nm} \mathcal{D} 1$ light. $\mathrm{Rb}-\mathrm{K}$ spin-exchange collisions polarize $\mathrm{K}$ vapor to $20 \%$ and $\mathrm{K}-{ }^{3} \mathrm{He}$ spin-exchange collisions polarize ${ }^{3} \mathrm{He}$ to $2 \%$. A droplet of alkali metal is used to block the stem of the cell and we position the droplet with independent temperature control of the cell and stem in order to minimize spin relaxation of ${ }^{3} \mathrm{He}$ due to dipolar fields created by its own magnetization.

The cell is heated in a boron nitride oven with GaP windows [34] using thick film resistive heaters driven by ac currents at $120 \mathrm{kHz}$. The oven is placed in an inner ferrite shield and three outer $\mu$-metal magnetic shields [35]. The diameter of the cell is a compromise between spin relaxation due to atomic diffusion to the walls, and the dimensions of the holes in the magnetic shields that limit magnetic shielding. In order to provide adequate thermal insulation and to reduce laser beam motion due to air convection, the cell, oven, and shields are placed in a vacuum chamber pumped to $<100 \mathrm{mTorr}$.

The pump light is generated by a Distributed Bragg Reflector laser amplified with a tapered amplifier (TA) and coupled into a single mode fiber. An accousto-optic modulator before the fiber allows pump intensity feedback while keeping the amplifier injection current constant. This approach eliminates laser pointing noise due to filamentation in the TA mode [36] and kinks in the TA's output power at high injection current densities [37].

The $x$ projection of K's spin, which constitutes the comagnetometer's signal, is measured via optical rotation of a linearly polarized probe beam that is blue detuned from $\mathrm{K}$ 's $\mathcal{D} 1$ line. The $11 \mathrm{~mW}$ probe beam is supplied by a DBR laser tuned to $769.53 \mathrm{~nm}$ and its plane of polarization is modulated with a photoelastic modulator and $\lambda / 4$ plate combination. The optical rotation signal is then extracted by demodulating the raw signal from the photodiode with a lock-in amplifier (see Fig. 1).

During the experiment, the comagnetometer is operated at the so-called compensation point where an applied $B_{z}$ field of about $3 \mathrm{mG}$ approximately cancels the field that $\mathrm{K}$ atoms experience from the magnetization of the ${ }^{3} \mathrm{He}$ spins, which results in strong coupling between $\mathrm{K}$ and ${ }^{3} \mathrm{He}$ spins. Residual magnetic fields inside the shields are canceled by coils within the ferrite shield and are adjusted

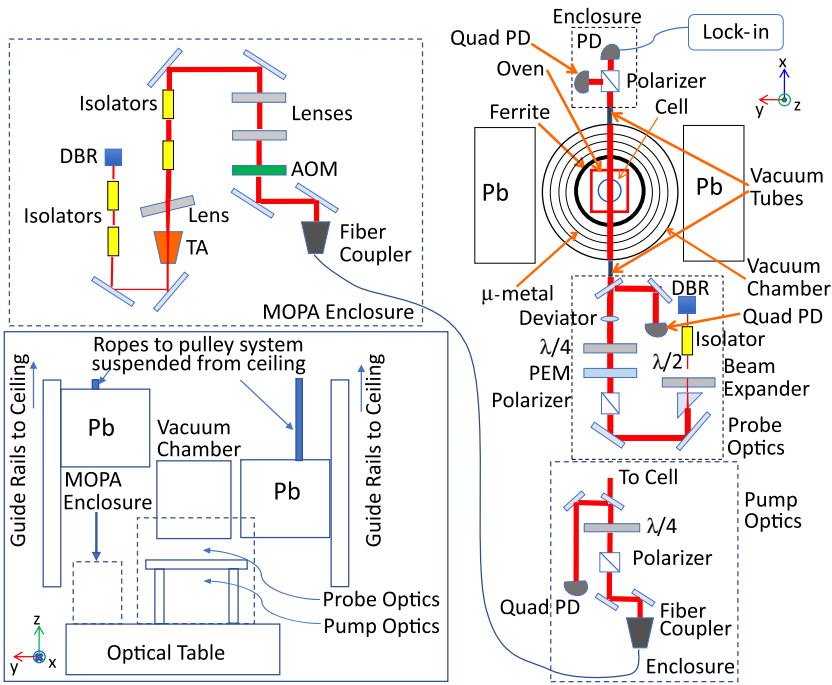

FIG. 1. Probe and pump optical setup in the $x-y$ plane. Bottom left insert: Experimental schematic in the $y-z$ plane.

by automated zeroing routines developed in [29]. Under these near zero-field and high alkali density conditions, $\mathrm{K}$ is in a spin-exchange relaxation free regime $[38,39]$ where the relaxation due to spin-exchange collisions is eliminated and high sensitivity is achieved. The signal of the comagnetometer at the compensation point is approximately given by

$$
S=\kappa \frac{P_{z}^{e} \gamma_{e}}{R_{\mathrm{tot}}^{e}}\left(\beta_{y}^{n}-\beta_{y}^{e}+\frac{\Omega_{y}}{\gamma_{n}}\right),
$$

where $\beta_{y}^{n}$ and $\beta_{y}^{e}$ are the anomalous magneticlike field couplings to the nuclear spin in ${ }^{3} \mathrm{He}$ and the electron spin in $\mathrm{K}$. For ordinary magnetic fields $\beta^{n}=\beta^{e}$, making the comagnetometer insensitive to magnetic fields to first order. $\Omega_{y}$ is the angular noninertial rotation rate of the apparatus about the $y$ axis. It represents an example of a nonmagnetic coupling to spin that does not cancel in the comagnetometer. $\gamma_{e}$ is the gyromagnetic ratio of the free electron while $\gamma_{n}$ is the gyromagnetic ratio of ${ }^{3} \mathrm{He} . P_{z}^{e}$ is the polarization of the $\mathrm{K}$ atoms and $R_{\mathrm{tot}}^{e}$ is their total spin relaxation rate; and $\kappa$ is a gain factor. The combination $\kappa P_{z}^{e} \gamma_{e} / R_{\text {tot }}^{e}$ serves as the calibration constant of the comagnetometer signal in magnetic field units. It can be measured using field modulation techniques by, for example, measuring the comagnetometer's response to a slow modulation of the $B_{x}$ field [40]. We verified the calibration independently by detecting a signal induced by a slow rotation $\Omega_{y}$ of the optical table, which was also measured with a tilt sensor.

To generate the anomalous spin-mass interaction we use two stacks of $\mathrm{Pb}$ bricks, with overall dimensions of $30 \mathrm{~cm} \times 36 \mathrm{~cm} \times 20 \mathrm{~cm}$ located along the $y$ axis of the comagnetometer (see Fig. 1). The face of the blocks is $13.6 \mathrm{~cm}$ from the center of the cell. The positions of the blocks are periodically reversed so that one of them is close to the cell while the other is raised by $50 \mathrm{~cm}$. This allows us to reverse the sign of the energy shift due to Eq. (1) to 
search for a correlation between the position of the masses and the comagnetometer's signal. The two $\mathrm{Pb}$ masses are suspended from the ceiling using ropes in such a way that the load on the ceiling is the same while they are at rest in the two positions. The weights are moved by a pulley system driven by a $4 \mathrm{~kW}$ servo motor connected to a reduction gear located close to the ceiling and far away from the comagnetometer. The mechanical system is constructed using nylon pulleys, fiber ropes, and aluminum frame and fasteners to minimize magnetic systematic effects. Furthermore, the dimensions of the system are designed so that the servo motor and reduction gear, which are magnetic, rotate an integer number of turns between the two positions and a $\mu$-metal shield is also placed over the motor and gear. The pump and probe optics are enclosed in nearly airtight plexiglass boxes and the laser beams propagate toward the vacuum can through sealed glass tubes to eliminate air convection due to motion of the masses. Thermal insulation is placed around the vacuum chamber to minimize mK-level temperature changes correlated with the position of the weights that resulted in nm-level relative mechanical motion of the comagnetometer components due to thermal expansion.

Considerable effort was also made to eliminate possible electromagnetic cross-talk between the power electronics of the motor and the comagnetometer. A separate computer controls the motor motion through an optically isolated interface. All equipment relating to the comagnetometer is powered from a different circuit from the motor's equipment. Electromagnetic interference arising from the high frequency switching supply in the motor's servo amplifier was reduced by placing filters on its input and output. Additional filtering was also employed on sensitive comagnetometer electronics and care was taken to eliminate any ground loops there. Lastly, the servo motor was programmed to turn off after the end of each motion before the data used in the correlation analysis were taken.

Results and discussion.-We collect data in records of 312 s after which the comagnetometer undergoes an automated sequence of zeroing routines. During each record, the weights are moved 31 times so that the starting configuration of the weights alternates after each zeroing. The weights are moved over $5 \mathrm{sec}$ and after a $3 \mathrm{sec}$ wait, data from the next $2 \mathrm{sec}$ are used in the analysis. A correlation "string" measurement [41] is formed by taking an appropriate weighted difference of the comagnetometer's signal. At the end of an individual run, which typically lasts around 2 days, the strings are combined in a weighted average to give a correlation measurement for that run. In the $\mathrm{K}-{ }^{3} \mathrm{He}$ comagnetometer the helicity of the pump light is fixed by the direction of the applied $B_{z}$ compensation field so that only two, $B+$ and $B-$, configurations are possible, which give opposite signs for possible spin-mass interactions. Figure 2 summarizes about 1.5 weeks of continuous data taking in both the $B+$ and $B-$ configurations. The reduced $\chi^{2}$ for these five runs is 0.73 .

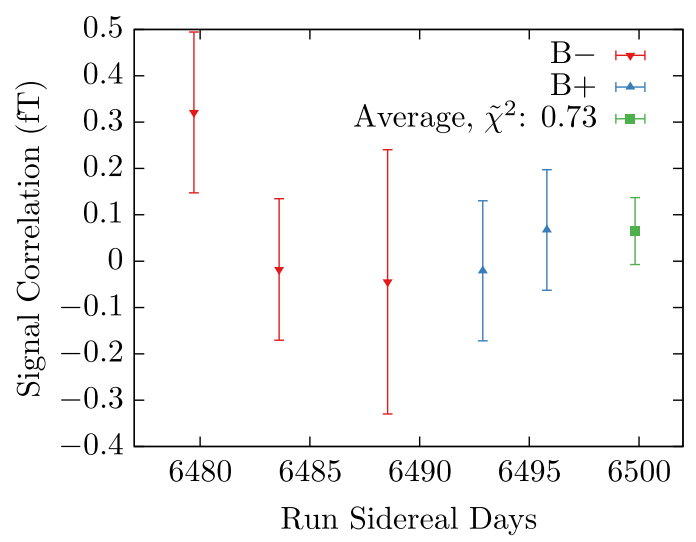

FIG. 2. Measured signal correlation. $B-$ runs are denoted with triangles pointing down while $B+$ runs are denoted with triangles pointing up. The weighted average is given by the square point. For 4 degrees of freedom, the $\tilde{\chi}^{2}$ is 0.73 .

Data from many other sensors are also collected in parallel and analyzed for correlations with the position of the weights. These include four-quadrant photodetectors for laser position measurements, noncontact distance sensors to measure small motion, a three-axis fluxgate magnetometer mounted outside the shields near the weights, and a two-axis tilt meter with nrad resolution mounted on the optical table.

Table I summarizes the results of these measurements of possible signal correlations. The sensors are calibrated by applying an appropriate excitation and measuring the main signal response and the sensor response. For example, the

TABLE I. Summary of measured correlations in the signal and other calibrated sensors.

\begin{tabular}{lc}
\hline \hline Sensor & Calibrated correlation (aT) \\
\hline Pump position $X$ & $2 \pm 4$ \\
Pump position $Y$ & $-12 \pm 7$ \\
Pump intensity & $2 \pm 5$ \\
Fluxgate $B_{x}$ & $-0.24 \pm 0.02$ \\
Fluxgate $B_{y}$ & $0.03 \pm 0.01$ \\
Fluxgate $B_{z}$ & $-0.19 \pm 0.03$ \\
Oven heater output & $-1 \pm 1$ \\
Vacuum can position & $-13 \pm 13$ \\
Pump glass tube position & $2 \pm 2$ \\
Probe position $X$ & $-12 \pm 9$ \\
Probe position $Y$ & $-3 \pm 6$ \\
Probe intensity & $-7 \pm 6$ \\
Rotation $\Omega_{x}$ & $1 \pm 1$ \\
Rotation $\Omega_{y}$ & $0.1 \pm 0.7$ \\
\hline Systematics total & $-41 \pm 20$ \\
\hline \hline Signal $(B+)$ & \\
Signal $(B-)$ & $-5 \pm 100$ \\
\hline Signal averaged & $70 \pm 100$ \\
\hline \hline
\end{tabular}




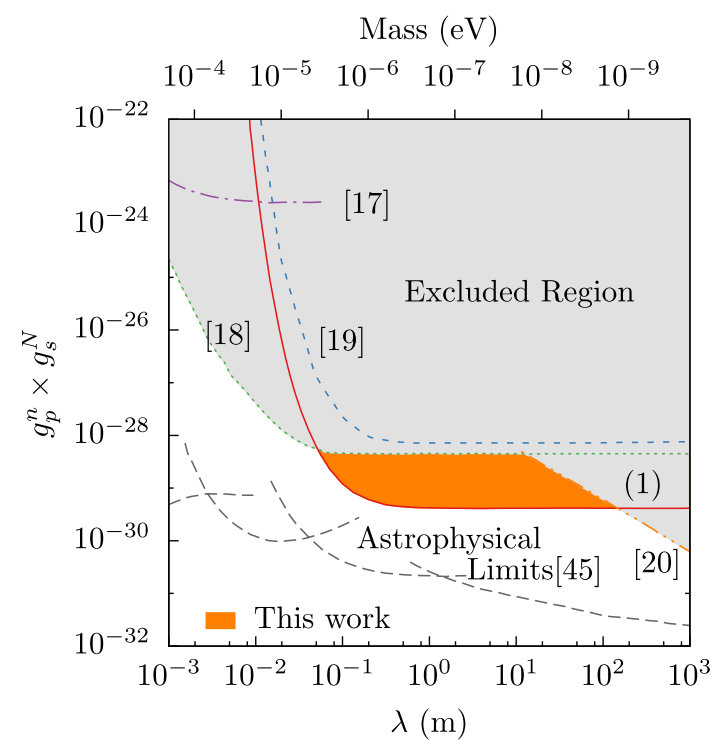

FIG. 3. Constraints (95\% C.L.) on $g_{p}^{n} g_{s}^{N}$ for the neutron. The solid red line (1) is from this work. Limits in [17,19] were multiplied by 2 to obtain a 95\% C.L. Reference [20] was extrapolated by integrating over the Earth's mass density for heavier axions. Reference [45] uses astrophysical constraints to limit $g_{p}^{n}$.

fluxgate field measurements are multiplied by the measured combined shielding factor of the magnetic shields and the comagnetometer compensation, which is $7 \times 10^{8}$ in the $x$ direction at the frequency pertinent to the experiment; other directions have higher suppressions.

We expect that systematic effects due to the sensor correlations are independent and their uncertainties can be combined in quadrature to provide an estimate of the overall systematic uncertainty. Since most correlations are not statistically significant, we do not correct the signal by the net sum of all measured correlations. Accordingly, we quote as the final result

$$
\beta^{n}-\beta^{e}<\left(32 \pm 70_{\text {stat }} \pm 20_{\text {syst }}\right) \text { aT },
$$

which gives an upper limit $\left|\beta^{e, n}\right|<155$ aT at $95 \%$ C.L.

As is typical, we assume that the scalar coupling to unpolarized fermions is the same for neutrons and protons and is 0 for electrons in the unpolarized mass so that this coupling reduces to $g_{s}^{N}$, the axion's scalar coupling to nucleons. Similarly, we denote the axion's pseudoscalar coupling to polarized neutrons and electrons as $g_{p}^{n}$ and $g_{p}^{e}$, respectively. In order to derive the constrains on $g_{p}^{n} g_{s}^{N}$, we note that the neutron is $87 \%$ polarized in ${ }^{3} \mathrm{He}[42,43]$ and we therefore set $\mu_{3 \mathrm{He}} \beta^{n}>g_{p}^{n} g_{s}^{N} 0.87 A$, where $A$ is the numerical factor from the integration of Eq. (1) over all the nucleons in the $\mathrm{Pb}$ masses. Similarly, we set $\mu_{B} \beta^{e}>$ $g_{p}^{e} g_{s}^{N} A$ to derive the limits on $g_{p}^{e} g_{s}^{N}$. Figures 3 and 4 show the respective constraints on $g_{p}^{n} g_{s}^{N}$ and $g_{p}^{e} g_{s}^{N}$. Our results set

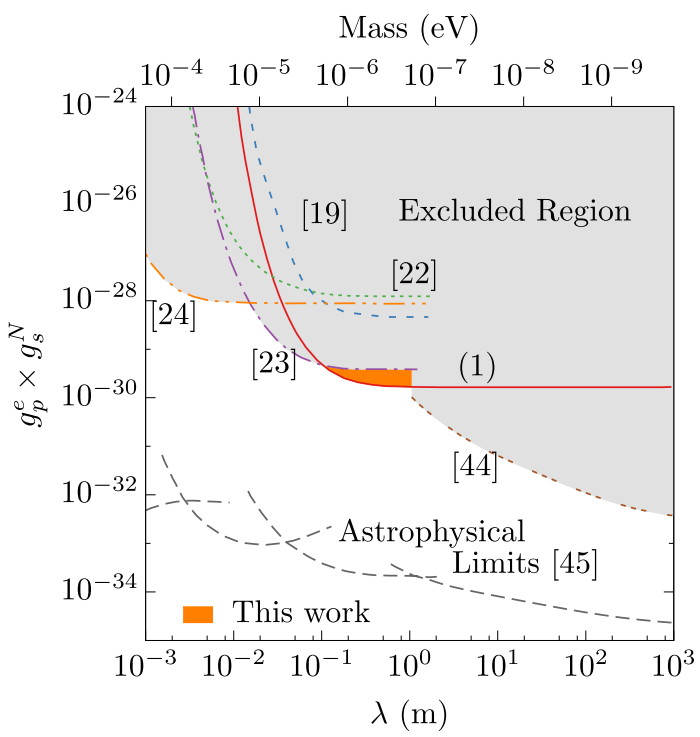

FIG. 4. Constraints (95\% C.L.) on $g_{p}^{e} g_{s}^{N}$ for the electron. The solid red line (1) is from this work. Limits in [19] were multiplied by 2 to obtain a 95\% C.L. Reference [45] uses astrophysical observations.

the most stringent laboratory limits for $m_{\phi}<3 \times 10^{-6} \mathrm{eV}$. For much lower axion masses additional constraints can be obtained from experiments using the Earth as the source mass $[20,44]$.

In conclusion, we have placed new laboratory constraints on $g_{p}^{n} g_{s}^{N}$ and $g_{p}^{e} g_{s}^{N}$ using a K-${ }^{3} \mathrm{He}$ comagnetometer and movable $\mathrm{Pb}$ masses. The limit on the neutron coupling is improved by an order of magnitude over 2 orders of magnitude in axion mass range. There is a significant potential for further improvement of the sensitivity since a similar comagnetometer spin force experiment has achieved a sensitivity of 1 aT [25]. The main challenge in the current experiment is due to mechanical motion of the masses instead of the electronic flip of the nuclear spins used in [25]. This causes subtle mechanical effects due to temperature changes correlated with the positions of the masses. Further improvement is possible by reducing the systematic sensitivity of the comagnetometer to such effects.

This work was supported by National Science Foundation Grant No. PHY-1404325.

*junyilee@princeton.edu

[1] S. Weinberg, A New Light Boson?, Phys. Rev. Lett. 40, 223 (1978).

[2] F. Wilczek, Problem of Strong $p$ and $t$ Invariance in the Presence of Instantons, Phys. Rev. Lett. 40, 279 (1978).

[3] R. D. Peccei and H. R. Quinn, cp Conservation in the Presence of Pseudoparticles, Phys. Rev. Lett. 38, 1440 (1977). 
[4] C. A. Baker, D. D. Doyle, P. Geltenbort, K. Green, M. G. D. vanderGrinten, P. G. Harris, P. Iaydjiev, S. N. Ivanov, D. J. R. May, J. M. Pendlebury, J. D. Richardson, D. Shiers, and K.F. Smith, Improved Experimental Limit on the Electric Dipole Moment of the Neutron, Phys. Rev. Lett. 97, 131801 (2006).

[5] B. Graner, Y. Chen, E. G. Lindahl, and B. R. Heckel, Reduced Limit on the Permanent Electric Dipole Moment of ${ }^{199} \mathrm{Hg}$, Phys. Rev. Lett. 116, 161601 (2016).

[6] J. E. Kim and G. Carosi, Axions and the strong $C P$ problem, Rev. Mod. Phys. 82, 557 (2010).

[7] E. Witten, Some properties of o(32) superstrings, Phys. Lett. 149B, 351 (1984).

[8] P. Svrcek and E. Witten, Axions in string theory, J. High Energy Phys. 06 (2006) 051.

[9] A. Arvanitaki, S. Dimopoulos, S. Dubovsky, N. Kaloper, and J. March-Russell, String axiverse, Phys. Rev. D 81, 123530 (2010).

[10] J.E. Moody and F. Wilczek, New macroscopic forces?, Phys. Rev. D 30, 130 (1984).

[11] P. Fayet, New interactions and the standard models, Classical Quantum Gravity 13, A19 (1996).

[12] B. A. Dobrescu and I. Mocioiu, Spin-dependent macroscopic forces from new particle exchange, J. High Energy Phys. 11 (2006) 005.

[13] R. Bradley, J. Clarke, D. Kinion, L. J Rosenberg, K. van Bibber, S. Matsuki, M. Mück, and P. Sikivie, Microwave cavity searches for dark-matter axions, Rev. Mod. Phys. 75, 777 (2003).

[14] CAST Collaboration, New cast limit on the axionphoton interaction, Nat. Phys. 13, 584 (2017).

[15] R. Ballou et al. (OSQAR Collaboration), New exclusion limits on scalar and pseudoscalar axionlike particles from light shining through a wall, Phys. Rev. D 92, 092002 (2015).

[16] E. G. Adelberger, B. R. Heckel, S. Hoedl, C. D. Hoyle, D. J. Kapner, and A. Upadhye, Particle-Physics Implications of a Recent Test of the Gravitational Inverse-Square Law, Phys. Rev. Lett. 98, 131104 (2007).

[17] M. Bulatowicz, R. Griffith, M. Larsen, J. Mirijanian, C. B. Fu, E. Smith, W. M. Snow, H. Yan, and T. G. Walker, Laboratory Search for a Long-Range $t$-Odd, $p$-Odd Interaction from Axionlike Particles using Dual-Species Nuclear Magnetic Resonance with Polarized ${ }^{129} \mathrm{Xe}$ and ${ }^{131} \mathrm{Xe}$ Gas, Phys. Rev. Lett. 111, 102001 (2013).

[18] K. Tullney et al., Constraints on Spin-Dependent ShortRange Interaction between Nucleons, Phys. Rev. Lett. 111, 100801 (2013).

[19] A. N. Youdin, D. Krause, Jr., K. Jagannathan, L. R. Hunter, and S. K. Lamoreaux, Limits on Spin-Mass Couplings within the Axion Window, Phys. Rev. Lett. 77, 2170 (1996).

[20] B. J. Venema, P. K. Majumder, S. K. Lamoreaux, B. R. Heckel, and E. N. Fortson, Search for a Coupling of the Earth's Gravitational Field to Nuclear Spins in Atomic Mercury, Phys. Rev. Lett. 68, 135 (1992).

[21] D. J. Wineland, J. J. Bollinger, D. J. Heinzen, W. M. Itano, and M. G. Raizen, Search for Anomalous Spin-Dependent Forces Using Stored-Ion Spectroscopy, Phys. Rev. Lett. 67, 1735 (1991).
[22] W.-T. Ni, S.-s. Pan, H.-C. Yeh, L.-S. Hou, and J. Wan, Search for an Axionlike Spin Coupling Using a Paramagnetic Salt with a dc Squid, Phys. Rev. Lett. 82, 2439 (1999).

[23] N. Crescini, C. Braggio, G. Carugno, P. Falferi, A. Ortolan, and G. Ruoso, Improved constraints on monopole-dipole interaction mediated by pseudoscalar bosons, Phys. Lett. B 773, 677 (2017).

[24] W. A. Terrano, E. G. Adelberger, J. G. Lee, and B. R. Heckel, Short-Range, Spin-Dependent Interactions of Electrons: A Probe for Exotic Pseudo-Goldstone Bosons, Phys. Rev. Lett. 115, 201801 (2015).

[25] G. Vasilakis, J. M. Brown, T. W. Kornack, and M. V. Romalis, Limits on New Long-Range Nuclear Spin-Dependent Forces Set with a K- ${ }^{3} \mathrm{He}$ Comagnetometer, Phys. Rev. Lett. 103, 261801 (2009).

[26] G. G. Raffelt, in Axions: Theory, Cosmology, and Experimental Searches, edited by M. Kuster, G. Raffelt, and B. Beltrán (Springer, Berlin, Heidelberg, 2008), pp. 51-71, https://doi.org/10.1007/978-3-540-73518-2_3.

[27] P. W. Graham, I. G. Irastorza, S. K. Lamoreaux, A. Lindner, and K. A. van Bibber, Experimental searches for the axion and axionlike particles, Annu. Rev. Nucl. Part. Sci. 65, 485 (2015).

[28] A. Arvanitaki and A. A. Geraci, Resonantly Detecting Axion-Mediated Forces with Nuclear Magnetic Resonance, Phys. Rev. Lett. 113, 161801 (2014).

[29] T. W. Kornack, R. K. Ghosh, and M. V. Romalis, Nuclear Spin Gyroscope Based on an Atomic Comagnetometer, Phys. Rev. Lett. 95, 230801 (2005).

[30] S. R. Schaefer, G. D. Cates, Ting-Ray Chien, D. Gonatas, W. Happer, and T. G. Walker, Frequency shifts of the magnetic-resonance spectrum of mixtures of nuclear spin-polarized noble gases and vapors of spinpolarized alkali-metal atoms, Phys. Rev. A 39, 5613 (1989).

[31] T. W. Kornack and M. V. Romalis, Dynamics of Two Overlapping Spin Ensembles Interacting by Spin Exchange, Phys. Rev. Lett. 89, 253002 (2002).

[32] E. Babcock, I. Nelson, S. Kadlecek, B. Driehuys, L. W. Anderson, F. W. Hersman, and T. G. Walker, Hybrid SpinExchange Optical Pumping of ${ }^{3} \mathrm{He}$, Phys. Rev. Lett. 91, 123003 (2003).

[33] M. V. Romalis, Hybrid Optical Pumping of Optically Dense Alkali-Metal Vapor without Quenching Gas, Phys. Rev. Lett. 105, 243001 (2010).

[34] N. Dural and M. V. Romalis, Gallium phosphide as a new material for anodically bonded atomic sensors, APL Mater. 2, 086101 (2014).

[35] T. W. Kornack, S. J. Smullin, S.-K. Lee, and M. V. Romalis, A low-noise ferrite magnetic shield, Appl. Phys. Lett. 90, 223501 (2007).

[36] L. Goldberg, M. R. Surette, and D. Mehuys, Filament formation in a tapered gaalas optical amplifier, Appl. Phys. Lett. 62, 2304 (1993).

[37] C. Fiebig, V.Z. Tronciu, M. Lichtner, K. Paschke, and $\mathrm{H}$. Wenzel, Experimental and numerical study of distributed-Bragg-reflector tapered lasers, Appl. Phys. B 99, 209 (2010). 
[38] W. Happer and H. Tang, Spin-Exchange Shift and Narrowing of Magnetic Resonance Lines in Optically Pumped Alkali Vapors, Phys. Rev. Lett. 31, 273 (1973).

[39] J. C. Allred, R. N. Lyman, T. W. Kornack, and M. V. Romalis, High-Sensitivity Atomic Magnetometer Unaffected by SpinExchange Relaxation, Phys. Rev. Lett. 89, 130801 (2002).

[40] J. M. Brown, S. J. Smullin, T. W. Kornack, and M. V. Romalis, New Limit on Lorentz- and $c p t$-Violating Neutron Spin Interactions, Phys. Rev. Lett. 105, 151604 (2010).

[41] W. B. Dress, P. D. Miller, J. M. Pendlebury, Paul Perrin, and Norman F. Ramsey, Search for an electric dipole moment of the neutron, Phys. Rev. D 15, 9 (1977).
[42] J. L. Friar, B. F. Gibson, G. L. Payne, A. M. Bernstein, and T. E. Chupp, Neutron polarization in polarized ${ }^{3} \mathrm{He}$ targets, Phys. Rev. C 42, 2310 (1990).

[43] J. J. Ethier and W. Melnitchouk, Comparative study of nuclear effects in polarized electron scattering from ${ }^{3} \mathrm{He}$, Phys. Rev. C 88, 054001 (2013).

[44] B. R. Heckel, E. G. Adelberger, C. E. Cramer, T. S. Cook, S. Schlamminger, and U. Schmidt, Preferred-frame and cp-violation tests with polarized electrons, Phys. Rev. D 78, 092006 (2008).

[45] G. Raffelt, Limits on a $C P$-violating scalar axion-nucleon interaction, Phys. Rev. D 86, 015001 (2012). 